\title{
Corrigendum
}

\section{A simple scheme for allocating capital in a foreign exchange proprietary trading firm}

\section{Antony Jackson}

Journal of Asset Management (2015) 16, 220. doi:10.1057/jam.2015.14

Correction to: Journal of Asset Management (2015) 16(1), 2-13.10.1057/jam2014.40

The author wishes to apologize to readers, and to thank Dr Florin Spinu for drawing to his attention some errors in the above article.

The following text addresses these errors, and should be read immediately after Equation (5) in the original article.

By using standard rules for manipulating expectations, variances and natural logs, the expression in Equation (5) simplifies to

$$
\begin{aligned}
\log \left(\mathbb{E}\left[W_{t+1}^{1-\gamma}\right]\right)= & (1-\gamma) \mathbb{E}\left[\log \left(W_{t+1}\right)\right] \\
& +\frac{1}{2}(1-\gamma)^{2} \mathbb{V}\left[\log \left(W_{t+1}\right)\right],
\end{aligned}
$$

and after dividing Equation (6) throughout by $(1-\gamma)$, we are left with the useful expression

$$
\begin{aligned}
\frac{1}{1-\gamma} \log \left(\mathbb{E}\left[W_{t+1}^{1-\gamma}\right]\right)= & \mathbb{E}\left[\log \left(W_{t+1}\right)\right] \\
& +\frac{1}{2}(1-\gamma) \mathbb{V}\left[\log \left(W_{t+1}\right)\right] .
\end{aligned}
$$

Applying natural logs to the budget equation, Equation (2), yields

$$
\log \left(W_{t+1}\right)=\log \left(1+R_{p, t+1}\right)+\log \left(W_{t}\right) .
$$

Substituting for $\log \left(W_{t+1}\right)$ from Equation (8) in Equation (7) yields the objective function

$$
\begin{aligned}
& \frac{1}{1-\gamma} \log \left(\mathbb{E}\left[W_{t+1}^{1-\gamma}\right]\right)=\mathbb{E}\left[\log \left(1+R_{p, t+1}\right)\right] \\
& +\mathbb{E}\left[\log \left(W_{t}\right)\right]+\frac{1}{2}(1-\gamma) \mathbb{V}\left[\log \left(1+R_{p, t+1}\right)\right] .
\end{aligned}
$$

Of course, $\mathbb{E}\left[\log \left(W_{t}\right)\right]$ is the same as $\log \left(W_{t}\right)$, because $W_{t}$ is known at time $t$. Constants do not affect the maximization exercise, so maximizing $(1) /(1-\gamma)$ $\log \left(\mathbb{E}\left[W_{t+1}^{1-\gamma}\right]\right)$ is equivalent to the following maximization problem:

$$
\begin{aligned}
\max \mathbb{E} & {\left[\log \left(1+R_{p, t+1}\right)\right] } \\
+ & \frac{1}{2}(1-\gamma) \mathbb{V}\left[\log \left(1+R_{p, t+1}\right)\right] .
\end{aligned}
$$

After introducing the lower case notation $r_{p},{ }_{t+1} \equiv \log \left(1+R_{p, t+1}\right)$, we arrive at the much simpler-looking maximization objective

$$
\max \mathbb{E}\left[r_{p, t+1}\right]+\frac{1}{2}(1-\gamma) \mathbb{V}\left[r_{p, t+1}\right]
$$

